community health workers with accessing care through referrals and transport provision.

Conclusion Accessing care for LBW infants in this community is a challenge. This will have a negative impact on the post-discharge outcomes of these vulnerable infants. There is an urgent need to develop strategies to address these barriers starting with the empowerment of women and their communities to accept and prioritise the care and survival of these infants in a context where their survival is often poor.

\section{G446(P) A SYSTEMATIC REVIEW EVALUATING THE METHODOLOGICAL QUALITY OF INFANT FORMULA TRIALS}

M Kunc, F Dewji, J Jobson, B Helfer, R Boyle. Paediatrics, Imperial College, London, UK

\subsection{6/archdischild-2020-rcpch.384}

Background Infant formula trials are carried out to assess safety and efficacy of infant formula, however an assessment of the risk of bias within these trials is lacking. Furthermore, it is currently unclear whether in these trials breastmilk feeding is appropriately supported.

Aims To assess the risk of bias in infant formula trials and establish whether it varies according to the human development index (HDI). The risk of undermining breastmilk feeding within the trial in relation to the human development index will also be examined.

Methods This systematic review carried out an investigation into controlled trials published from $1 / 1 / 16$ to $17 / 2 / 19$, using the Cochrane Risk of Bias 2.0 tool, as well as analysing the risk of undermining breastmilk feeding using signalling questions derived from a previous Delphi consensus. Included trials had a population under 3 years old, where one infant formula was compared against another infant formula with modified ingredients.

Results This study considered 44 main trial publications, where $61 \%$ had a high overall risk of bias. In individual domains, highest risk of bias was identified in deviation from intended intervention (36\%) and selective outcome reporting (18\%). There was no significant association between HDI and risk of bias in any domain $(p>0.05)$. A high risk of undermining lactation tended to be present in lower HDI countries (OR 0.29 , 95\% CI 0.80 to 1.08 , $\mathrm{p}=0.06) .70 \%$ of the trials overall and $63 \%$ of those with high risk of undermining lactation were funded by BMS industry.

Conclusion These findings suggest a possible relationship between risk of undermining lactation and HDI, which needs to be explored further. No relationship between risk of bias and HDI was found. Further breastfeeding protection and promotion worldwide is needed.

\section{G447(P) IAP IMMUNIZE INDIA: SMS TEXT BASED VACCINATION REMINDER SERVICE PROGRAM - AN INNOVATION}

RK Pejaver. Neonatology, People tree @ Meenakshi Hospitals, Bangalore, India

10.1136/archdischild-2020-rcpch.385

Objectives To Improve vaccine uptake and compliance in Childhood vaccine program by sending text alerts on phone.
Methods A soft ware program was created with a national sort code. Any person in India can register their child by sending an SMS with the child's name and date of birth to 566778 from their mobile phone. For example: 'Immunize $>$ name $<$ date of birth'. The same phone will then receive vaccination reminders for 12 years, whenever the child is due for a shot. Three reminders, two before the due date in that week and one after the due date. It is across all mobile networks anywhere in India. It is free of cost to the client.

Immunize India Charities then partnered with Indian Academy of Pediatrics (IAP)to endorse the program. Indian academy of Pediatrics professional organization, with over 30000 members. IAP is the disseminator and motivator. Immunize India Charities are continuing to do the the back end work of the program. There is an option of joining the program for children who are already into their vaccination schedule. There is an option for a family to opt out of the program.

Results Pilot program tested before the launch. Impact value is $11 \%$ increase in uptake. The program currently has more than 1.7 million registrations.

Conclusions Very effective. Can be done in any language or multiple languages.

Utilizes the most popular cell phone and text modality.

Software based program and hence limited human resource required to implement. Soft ware is very strong. All messages are automatically generated and proof of sending the message can be had.

Can be scaled up with ease. Now the program also has an app.

The program was sought by University of Kentucky, USA. Implemented it as a pilot program in the city of Kentucky. Immunize India translated it into Spanish. The impact of the program showed improvement in the compliance and uptake of immunization. 18\% increase in compliance in the 6 month cohort in the subgroup of under privileged part of the society.

Immunize India is ready to offer the program to any interested government or non profit organization, provide guidance to implement.

\section{G448(P) QUALITY OF CARE DATA COLLECTION IN SIERRA LEONE - IMPORTANCE OF NARRATIVE AND CONTEXT}

S Blakey. Global Team, Royal College of Paediatrics and Child Health, London, UK

\subsection{6/archdischild-2020-rcpch.386}

Background Emergency Triage, Assessment and Treatment plus $($ ETAT +$)$ was adapted as a quality improvement intervention and rolled out to regional and district hospitals in Sierra Leone in 2017 and 2018. UK mentors partnering with ETAT + -trained Sierra Leonean nurse-mentors were placed in participating facilities for 6 months to provide a 3-month teaching programme with 3 months' follow-up clinical support. Data were collected on Quality of Care (QOC) including management of severe respiratory distress (SRD) and prescription of key medications and blood transfusions, selected as core aspects of ETAT+. Aggregate data showed improvements in QOC over the two intervention periods. ETAT + is now part of Sierra Leone's national health strengthening strategy. In 2019, a 3-year ETAT + programme was initiated in all government hospitals. 
Methods In 2019, programme mentors identified factors outside the control of the intervention potentially affecting performance as reflected in the QoC data, including lack of oxygen concentrators, electricity and essential medicines. Alongside QOC measures, narrative data were recorded to test this. QOC data from one four-week period (May 2019) in four 'hub' regional hospitals were assessed against qualitative data. Frequency of UK mentor intervention was also recorded. Results Variability in QoC between hospitals corresponded with narrative findings. While 3 hubs showed 100\% correct oxygen prescription for SRD, one recorded 33\% corresponding with narrative reporting of issues with oxygen concentrators and electricity supply. The same hub recorded $8 \%$ appropriate blood transfusions, with narrative reporting of prescriptions managed by a practitioner without ETAT + experience; the other hubs reported 100\%. Need for external mentors to intervene e.g. for management of critically unwell children and intravenous cannulation varied from 20-79\%; a higher frequency of mentor intervention corresponded with higher QoC scores.

Discussion ETAT+ plausibly improves QOC in participating facilities in Sierra Leone. However it is important to observe factors outside of the intervention's direct control which may negatively affect results. Staff knowledge and performance may improve but remain constrained by poor equipment and infrastructure. Presence of external programme clinical mentors may affect results (with thresholds to intervene varying between clinicians). More detailed understanding of the hospital care 'ecology' is required to assess ETAT+'s impact on care quality and health outcomes.

\section{G449(P) USING THE MODIFIED DELPHI METHOD TO ESTABLISH CONSENSUS ON CLINICAL ALGORITHMS FOR A NEONATAL MOBILE HEALTH APPLICATION FOR LOW- INCOME COUNTRIES}

${ }^{1} \mathrm{M}$ Evans, ${ }^{1} \mathrm{C} C$ Crehan, ${ }^{2,3} \mathrm{M}$ Corden, ${ }^{1,4} \mathrm{M}$ Heys. ${ }^{1}$ Great Ormond Street Hospital Institute of Child Health, UCL, London, UK; ${ }^{2}$ Division of Hospital Medicine, Department of Pediatrics, Children's Hospital Los Angeles, Los Angeles, USA; ${ }^{3}$ Pediatrics, Keck School of Medicine, University of Southern California, LOS Angeles, USA; ${ }^{4}$ Specialist Children's and Young People's Services, East London NHS FT, London, UK

\subsection{6/archdischild-2020-rcpch.387}

Aim A neonatal m-health application (NeoTree) is being codesigned to support health care workers (HCWs) in lowincome countries (LICs). A key function is the digital implementation of evidence-based guidelines. Clinical support algorithms have been developed using best available national and international evidence and clinical judgement. We aimed to determine whether a panel of neonatal experts could address evidence gaps by reaching a consensus on four algorithms: neonatal sepsis, birth asphyxia, respiratory distress and hypothermia.

Methods This was a consensus-generating study using a twostep modified Delphi method. In round one, experts rated algorithm items and justified their responses. Items meeting consensus ( $>80 \%$ agreement) were included. Items not meeting consensus were excluded or only included following major edits or unchanged if they presented core elements of evidence-based guidelines. In round two, experts further rated items from round one that did not reach consensus.

Results Fourteen experts participated in round one, ten in round two. Nine were from high-income countries, five from
LICs. Experts included physicians and nurse practitioners with an average neonatal experience of 20 years, 12 in LICs. After two rounds, a consensus was reached on $52 \%$ of 84 items. Experts consistently stated that items must be consistent with local and WHO guidelines. An example of a key change in an algorithm included the management of transient tachypnoea of the newborn (TTN). The proposed NeoTree algorithm aimed to differentiate TTN from other respiratory conditions to avoid unnecessary antibiotic use. Expert consensus was that HCWs would be unable to appropriately distinguish and monitor patients with TTN. In the revised respiratory algorithm, all neonates with respiratory distress will be given antibiotics. As a result, the final algorithms included $53(62 \%)$ items.

Conclusion Four algorithms in a neonatal m-health treatment app were reviewed and refined by consensus expert opinion. Revisions to the NeoTree app were made in response to these findings and will be clinically validated in an imminent study. Expert discussion reinforced gaps in evidence in neonatal care in LIC, highlighting areas for future research.

\section{G450(P) SHORT TERM GLOBAL HEALTH PROJECT: A PILOT SCHEME IN SIERRA LEONE TO ENHANCE TRAINEE DEVELOPMENT}

${ }^{1}$ A Zahari, ${ }^{1} T$ Oliver, ${ }^{1} \mathrm{~N}$ Kennedy, ${ }^{2} \mathrm{R}$ Emeric, ${ }^{3} \mathrm{P}$ Turay, ${ }^{1} \mathrm{~V}$ Sawicki, ${ }^{4} \mathrm{P}$ Chahal, ${ }^{1,5} \mathrm{P}$ Chahal. ${ }^{1}$ Postgraduate Training, Health Education East Midlands, Leicester, UK; ${ }^{2}$ Public Health, University of Makeni, Makeni, Sierra Leone; ${ }^{3}$ Chief Medical Officer, Holy Spirit Hospital, Makeni, Sierra Leone; ${ }^{4}$ Co-founder, Help Madina, UK; ${ }^{5}$ Associate Professor, University of Nottingham, Nottingham, UK

\subsection{6/archdischild-2020-rcpch.388}

Introduction The NHS is experiencing declining number of doctors entering specialist training. Additionally, the BMA reports travel as the main reason for taking time out of training. The Global Health Qualitative Evaluation Project $(\mathrm{GH}-$ QEP) is an innovative pilot scheme that aims to address this. We believe opportunities to work abroad for a short term within a training program will appeal to those considering leaving the NHS or as an alternative to time out of program. It also builds skills and understanding, enhancing long term development as clinicians.

Design GH-QEP was a 4-week program based in Sierra Leone. Our team comprised of one paediatric and two GP trainees from the UK. Senior support was provided by UK and local consultants. The project consisted of qualitative evaluation of the Sierra Leone healthcare system post-Ebola through interviews of healthcare professionals and the public. The Ebola epidemic exposed an already stretched healthcare system within the country creating a climate of uncertainty. Significant effort implemented by the government and private organisations looked to rectify this. We received training in qualitative research methodology, and led the project design and implementation. Observing clinical practice in a variety of settings (hospital, rural feeding clinic, and district health unit) allowed hands-on experience of health issues in a challenging environment.

Outcome Undertaking qualitative evaluation is an opportunity not routinely provided in UK specialty training programs. Through patient interviews we learnt about the impact of government policies, education, local health beliefs and priorities of rural and urban population. It allowed us to reflect on the assumptions which shape our own understandings of health, 